\title{
Golden SusPtrit: a genetically well transformable barley line for studies on the resistance to rust fungi
}

\author{
F. K. S. Yeo • G. Hensel · T. Vozábová • A. Martin-Sanz • \\ T. C. Marcel · J. Kumlehn · R. E. Niks
}

Received: 13 August 2013 / Accepted: 18 October 2013

(C) Springer-Verlag Berlin Heidelberg 2013

\begin{abstract}
Key message We developed 'Golden SusPtrit', i.e., a barley line combining SusPtrit's high susceptibility to non-adapted rust fungi with the high amenability of Golden Promise for transformation.

Abstract Nonhost and partial resistance to Puccinia rust fungi in barley are polygenically inherited. These types of resistance are principally prehaustorial, show high diversity between accessions of the plant species and are genetically associated. To study nonhost and partial resistance, as well as their association, candidate gene(s) for resistance must be cloned and tested in susceptible material where SusPtrit would be the line of choice. Unfortunately, SusPtrit is not amenable to Agrobacterium-mediated transformation.
\end{abstract}

Communicated by P. M. Hayes.

Electronic supplementary material The online version of this article (doi:10.1007/s00122-013-2221-7) contains supplementary material, which is available to authorized users.

\section{F. K. S. Yeo}

Department of Plant Science and Environmental Ecology, Faculty of Resource Science and Technology, University Malaysia

Sarawak, 94300 Kota Samarahan, Sarawak, Malaysia

G. Hensel $\cdot$ J. Kumlehn

Leibniz Institute of Plant Genetics and Crop Plant

Research (IPK), Plant Reproductive Biology, Corrensstr. 3,

06466 Gatersleben, Germany

T. Vozábová · A. Martin-Sanz · T. C. Marcel · R. E. Niks ( $₫)$

Laboratory of Plant Breeding, Wageningen University, 6700

AJ Wageningen, The Netherlands

e-mail: rients.niks@wur.nl

T. C. Marcel

INRA-AgroParisTech, UR1290 BIOGER-CPP, Avenue Lucien

Brétignières, BP01, 78850 Thiverval-Grignon, France
Therefore, a doubled haploid (DH) mapping population $(n=122)$ was created by crossing SusPtrit with Golden Promise to develop a 'Golden SusPtrit', i.e., a barley line combining SusPtrit's high susceptibility to non-adapted rust fungi with the high amenability of Golden Promise for transformation. We identified nine genomic regions occupied by resistance quantitative trait loci (QTLs) against four non-adapted rust fungi and $P$. hordei isolate 1.2.1 (Ph.1.2.1). Four DHs were selected for an Agrobacterium-mediated transformation efficiency test. They were among the $12 \mathrm{DH}$ lines most susceptible to the tested nonadapted rust fungi. The most efficiently transformed $\mathrm{DH}$ line was SG062N (11-17 transformants per 100 immature embryos). The level of non-adapted rust infection on SG062N is either similar to or higher than the level of infection on SusPtrit. Against Ph.1.2.1, the latency period conferred by SG062N is as short as that conferred by SusPtrit. SG062N, designated 'Golden SusPtrit', will be a valuable experimental line that could replace SusPtrit in nonhost and partial resistance studies, especially for stable transformation using candidate genes that may be involved in rustresistance mechanisms.

\section{Introduction}

Nonhost resistance implies immunity of all members of a plant species against a potential pathogen species (Niks et al. 2011). The resistant plant species is referred to as nonhost, and the would-be pathogen species is referred to as heterologous or non-adapted pathogen. Classification of a plant species as nonhost or host against certain potential pathogen species is not easy (Niks 1987; Niks et al. 2011). Some plant species have few accessions with an intermediate level of susceptibility to a particular pathogen. Such 